\title{
MULTIPLICATION IN GROTHENDIECK RINGS OF INTEGRAL GROUP RINGS
}

BY D. L. STANCL

Communicated by I. Reiner, June 24, 1966

1. Introduction. Let $G$ be a finite group, $Z$ the ring of rational integers, and form the Grothendieck ring $K^{0}(Z G)$ of the integral group ring $Z G$. Swan [4] has described multiplication in $K^{0}(Z G)$ when $G$ is cyclic of prime power order. The purpose of this note is to present results which describe multiplication in $K^{0}(Z G)$ when $G$ is cyclic or elementary abelian. Full details will appear elsewhere.

Let $Q$ denote the rational field, and recall that the elements of $K^{0}(Q G)$ are $Z$-linear combinations of symbols $\left[M^{*}\right]$, where $M^{*}$ ranges over all finitely-generated left $Q G$-modules, and similarly for $K^{0}(Z G)$. We define a ring epimorphism $\theta: K^{0}(Z G) \rightarrow K^{0}(Q G)$ by $\theta[M]$ $=\left[Q \otimes_{Z} M\right]$, and call any linear mapping $f: K^{0}(Q G) \rightarrow K^{0}(Z G)$ such that $\theta f=1$ a lifting map for $K^{0}(Z G)$. Since the Jordan-Hölder Theorem holds for $Q G$-modules, $K^{0}(Q G)$ is the free abelian group with basis $\left\{\left[M_{i}^{*}\right]: 1 \leqq i \leqq m\right\}$, where $\left\{M_{i}^{*}: 1 \leqq i \leqq m\right\}$ is a full set of nonisomorphic irreducible $Q G$-modules. Swan [4] has shown that to describe multiplication in $K^{0}(Z G)$ it suffices to describe the products $f\left[M_{i}^{*}\right] \cdot f\left[M_{j}^{*}\right]$, for $1 \leqq i, j \leqq m$, and $f\left[M_{i}^{*}\right] x$, for $1 \leqq i \leqq m$ and $x \in \operatorname{ker} \theta$.

2. Statement of results. Let $G$ be cyclic of order $n$ with generator g. For each $s$ dividing $n, \zeta_{s}$ will denote a primitive $s$ th root of unity, and $Z_{s}$ will denote the $Z G$-module $Z\left[\zeta_{s}\right]$ on which $g$ acts as $\zeta_{s}$. Similarly, $Q_{s}$ will denote the $Q G$-module $Q\left(\zeta_{s}\right)$. Then $K^{0}(Q G)$ is the free abelian group with basis $\left\{\left[Q_{s}\right]: s \mid n\right\}$, and $f: K^{0}(Q G) \rightarrow K^{0}(Z G)$ by $f\left[Q_{s}\right]=\left[Z_{s}\right]$ is a lifting map. Swan [4] has shown that $f$ is a ring homomorphism. Also, for each $s$ dividing $n, G_{s}$ will denote the quotient group of $G$ of order $s$, and if $t \mid s, N_{s / t}$ will denote the norm from $Q_{s}$ to $Q_{t}$. By the results of Heller and Reiner [2],

$$
\operatorname{ker} \theta=\left\{\sum_{s \mid n}\left(\left[A_{s}\right]-\left[Z_{s}\right]\right): A_{s}=Z_{s} \text {-ideal in } Q_{s}\right\} .
$$

Theorem 1. Multiplication in $K^{\circ}(Z G)$ is given by the formula

$$
\left[Z G_{r}\right]\left(\left[A_{s}\right]-\left[Z_{s}\right]\right)=\sum_{d}\left(\left[N_{s / s^{\prime}}\left(A_{s}\right) Z_{d}\right]-\left[Z_{d}\right]\right),
$$

for all $r$, s dividing $n$, where $s^{\prime}=s /(r, s)$ and $d$ ranges over all divisors of $[r, s]$ such that $\left([r, s] / d, s^{\prime}\right)=1$. 
THEOREM 2. If $G$ is an elementary abelian group, multiplication in $K^{0}(Z G)$ can be explicitly determined.

We remark that it is possible to give formulas which describe multiplication in $K^{0}(Z G)$ when $G$ is elementary abelian. These formulas will not be included here.

3. Proof of Theorem 1. We first suppose that $r=p^{a}$, for some prime $p$ and nonnegative integer $a$, and write $s=p^{b_{t}},(p, t)=1$. If $a=0$ or $b=0$, the theorem is trivial. Let $\hat{Z}=Z_{s} / A_{s}$ and for each $t$ dividing $s$, let $\hat{Z}\left\langle\bar{\zeta}_{t}\right\rangle$ denote the $Z G$-module $\hat{Z}$ on which $g$ acts as $\zeta_{t}$ reduced modulo $A_{8}$. It suffices to find $M=Z G_{p^{a}} \otimes_{z} \hat{Z}$. Since $Z G_{p^{a}} \cong Z[x] /\left(x^{p^{a}}-1\right)$, $M \cong \hat{Z}[x] /\left(x^{p^{a}}-1\right)$. If $a \leqq b$, then in $\hat{Z}[x], x^{p^{a}}-1=\prod_{k}\left(x-\xi_{p^{a}}^{k}\right)$, $1 \leqq k \leqq p^{a}$, and thus $M \cong \sum_{k} \hat{Z}\left\langle\xi_{p}^{b} \xi_{p}^{k_{a}}\right\rangle$. A calculation with norms now yields the desired result. If $a>b$, then $x^{p a}-1$ factors in $\hat{Z}[x]$ as follows: $x^{p a}-1=\prod_{k}\left(x-\xi_{p^{b}}^{k}\right) \prod_{i, j}\left(x^{p^{i-b}}-\xi_{p^{b}}^{j}\right)$, where $1 \leqq k \leqq p^{b}, b+1$ $\leqq i \leqq a$, and $1 \leqq j \leqq p^{b}$ with $(p, j)=1$. Therefore

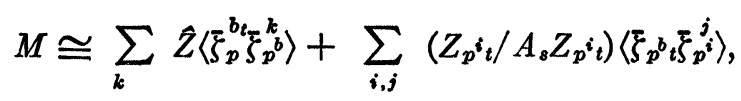

where $\left(Z_{p^{i} t} / A_{s} Z_{p^{i} t}\right)\left\langle\xi_{p}^{b_{t}} \xi_{p^{j_{i}}}\right\rangle$ denotes the $Z G$-module $Z_{p^{i} t} / A_{s} Z_{p^{i} t}$ on which $g$ acts as $\xi_{p}^{b} \xi_{p}^{j_{i}}$. Again, a calculation with norms will yield the desired result. This proves the theorem for the case $r=p^{a}$. The general case follows by the use of induction on the number of distinct prime divisors of $r$.

4. Proof of Theorem 2. In order to prove Theorem 2, we need several lemmas.

LEMMA 1. Let $G$ be an abelian group, $F$ an algebraic number field which is a splitting field for $G$, and $R$ the ring of algebraic integers of $F$. Then multiplication in $K^{0}(R G)$ can be explicitly determined.

Let $G$ be an elementary abelian group and write $G=G_{1} \times \cdots \times G_{k}$, where $G_{i}$ is cyclic of order $p$ with generator $g_{i}$, for $1 \leqq i \leqq k$. Let $\zeta$ be a primitive $p$ th root of unity, $F=Q(\zeta), R=Z[\zeta]$, and denote by $F\left\langle a_{1}, \cdots, a_{k}\right\rangle$ the $F G$-module $F$ on which $g_{i}$ acts as $\zeta^{a_{i}}$, where $1 \leqq a_{i} \leqq p$ for $1 \leqq i \leqq k$. Similarly, if $A$ is any $R$-ideal in $F, A\left\langle a_{1}, \cdots, a_{k}\right\rangle$ will denote the $R G$-module $A$ on which $g_{i}$ acts as $\xi^{a_{i}}$. Note that, by restriction of operators, $F\left\langle a_{1}, \cdots, a_{k}\right\rangle$ and $A\left\langle a_{1}, \cdots, a_{k}\right\rangle$ are $Q G$ and $Z G$-modules, respectively. It is easy to prove that the $Q G$ modules of form $F\left\langle p, \cdots, p, 1, a_{j+1}, \cdots, a_{k}\right\rangle$, where $1 \leqq j \leqq k$, together with the trivial module $Q$, form a full set of nonisomorphic irreducible $Q G$-modules.

Define $\psi: K^{0}(Z G) \rightarrow K^{0}(R G)$ by $\psi[Y]=\left[R \otimes_{z} Y\right]$, where $R \otimes_{z} Y$ is an $R G$-module with action of $R$ given by $r^{\prime}(r \otimes y)=r^{\prime} r \otimes y$, for all 
$r^{\prime} \in R$, and action of $G$ given by $g(r \otimes y)=r \otimes g y$, for all $g \in G$. Similarly, define $\eta: K^{0}(Q G) \rightarrow K^{0}(F G)$ by $\eta\left[Y^{*}\right]=\left[F \otimes_{Q} Y^{*}\right]$.

LEMMA $2 . \psi$ and $\eta$ are ring homomorphisms and the following diagram commutes and is exact:

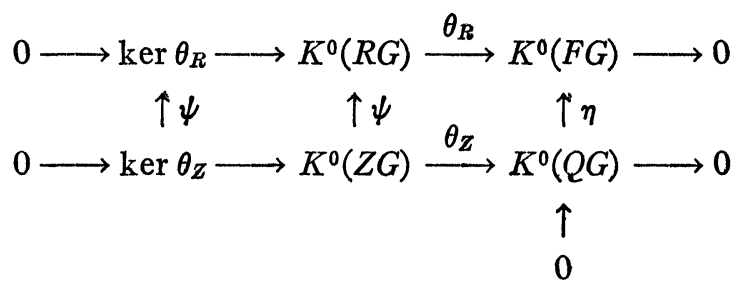

Let $\Phi_{p}(x)$ denote the cyclotomic polynomial of order $p$. If we apply $\psi$ to $\left[A\left\langle p, \cdots, p, 1, a_{j+1}, \cdots, a_{k}\right\rangle\right] \in K^{0}(Z G)$, we note that $\Phi_{p}\left(g_{j}\right)$ annihilates $R \otimes_{z} A\left\langle p, \cdots, p, 1, a_{j+1}, \cdots, a_{k}\right\rangle$. Since $\Phi_{p}(x)$ splits into linear factors in $R[x]$, this gives us a composition series for $R \otimes_{z} A\left\langle p, \cdots, p, 1, a_{j+1}, \cdots, a_{k}\right\rangle$. If we denote by $A^{(t)}$ the ideal conjugate to $A$ under the $Q$-automorphism of $F$ which takes $\zeta$ into $\zeta^{t}$, we thus obtain

Lemma 3. $\psi\left[A\left\langle p, \cdots, p, 1, a_{j+1}, \cdots, a_{k}\right\rangle\right]=\sum_{t}\left[A^{(t)}\langle p, \cdots\right.$, $\left.\left.p, t, t a_{j+1}, \cdots, t a_{k}\right\rangle\right]$, where $1 \leqq t \leqq p-1$.

We now use the formulas for $\operatorname{ker} \theta_{z}$ and $\operatorname{ker} \theta_{R}$ obtained by Heller and Reiner [2], and our formula for $\psi\left[A\left\langle p, \cdots, p, 1, a_{j+1}, \cdots, a_{k}\right\rangle\right]$, to show that $\psi: \operatorname{ker} \theta_{Z} \rightarrow \operatorname{ker} \theta_{R}$ is monic. Lemma 2 then implies that $\psi: K^{0}(Z G) \rightarrow K^{0}(R G)$ is monic. Now define $f_{R}: K^{0}(F G) \rightarrow K^{0}(R G)$ by $f_{R}\left[F\left\langle a_{1}, \cdots, a_{k}\right\rangle\right]=\left[R\left\langle a_{1}, \cdots, a_{k}\right\rangle\right]$. It is clear that $f_{R}$ is a lifting map for $K^{0}(R G)$, and it is easy to show that $f_{R}$ is a ring homomorphism. Since $\psi$ is monic, we may define $f_{Z}=\psi^{-1} f_{R} \eta$. Then $f_{Z}$ is a lifting map for $K^{0}(Z G)$ and is a ring homomorphism. Finally, since $F$ is a splitting field for $G$, multiplication in $K^{0}(R G)$ is known by Lemma 1, and hence multiplication in $K^{0}(Z G)$ can be explicitly determined by the use of the monomorphism $\psi$. This completes the proof of Theorem 2.

\section{BIBLIOGRAPHY}

1. A. Heller and I. Reiner, Grothendieck groups of orders in semisimple algebras, Trans. Amer. Math. Soc. 112 (1964), 344-355.

2. - Grothendieck groups of integral group rings, Illinois J. Math. 9 (1965), 349-359.

3. R. G. Swan, Induced representations and projective modules, Ann. of Math. (2), 71 (1960), 552-578.

4. - The Grothendieck ring of a finite group, Topology 2 (1963), 85-110.

UNIVERSITY OF ILLINOIS 